\title{
Characterization of extinction/reignition events in turbulent premixed counterflow flames using strain-rate analysis
}

\author{
Ranjith R. Tirunagari ${ }^{\mathrm{a}, *}$, Stephen B. Pope ${ }^{\mathrm{a}}$ \\ ${ }^{a}$ Sibley School of Mechanical and Aerospace Engineering, Cornell University, Ithaca, NY 14853, USA \\ Colloquium choice: Turbulent Flames
}

Manuscript Number: PROCI-D-15-00188

Two-column version: page 1: 140, pages 2-8: 6300, total count is 6440 words, revised limit is 6200 words

Two-column version: page 1: 0.16 , pages 2-8: 7, total length is $7-1 / 6$ pages, length limit is $7-1 / 3$ pages

\begin{abstract}
We investigate extinction/reignition events in two contrasting turbulent premixed flames of the Yale turbulent counterflow flame (TCF) burner, that are both qualitatively and quantitatively different. One of the two chosen flames is a high-burning (HB) flame with a low probability of local extinction while the other flame is a low-burning (LB) flame with a high probability of local extinction. In recent work, we successfully studied the turbulent premixed flames of the Yale TCF burner using the large-eddy simulation/probability density function (LES/PDF) methods. In this present study, the main motivation is to investigate how the compositional structure of the two turbulent flames are related to that of laminar flames. To this end, steady, one-dimensional, strained, opposed-jet laminar flame calculations are performed to investigate the effect of strain rates $K$ on the laminar counterparts of $\mathrm{HB}$ and LB, and to evaluate the extinction strain rates $S_{\text {ext }}$ for the two flames. Subsequently, a normalized distance $Z$ is defined in terms of the mixture fraction $\xi$, which is calculated based on the mass fraction of $N_{2}$. The scatter plots from the particle data of (a) $\mathrm{CH}_{2} \mathrm{O}$ mass fraction $\mathrm{Y}_{\mathrm{CH}_{2} \mathrm{O}}^{*}$ vs. progress variable $p^{*}$ and (b) temperature $T^{*} v s$. the normalized distance $Z^{*}$ are quite different for the two flames with more samples close to the extinguished laminar profile for the LB flame than for the HB flame. The cell-mean profiles of $T$ vs. $Z$ resemble the laminar profiles at different strain rates even though the LES/PDF are non-trivially 3D and unsteady. These cell-mean profiles are used to evaluate the instantaneous equivalent steady strain rate (ESSR) $S$ for the two flames. The cumulative distribution function (CDF) of $S$, conditional on $S<S_{\text {ext }}$ (i.e., burning samples), is somewhat similar, with the distributions being broad without a peak close to the bulk strain rate. However, comparatively, more samples of the LB flame have the ESSR values above the extinction strain rate, i.e., $S>S_{\text {ext }}$. The scatter
\end{abstract}


plots of the ESSR $S$ vs. the fresh product layer thickness $\Delta_{f}$ quantify the thinning of the product layer as the strain rate $S$ increases. The scatter for the HB flame follow the corresponding laminar profile quite closely, whereas, the thicknesses observed for the LB flame are higher compared to its laminar prediction. Keywords: Large-eddy simulation, Probability density function methods, Turbulent premixed counterflow flames, Extinction/reignition, Strain rate

\section{Introduction}

Turbulent counterflow flames (TCFs) have garnered significant attention in recent years in the combustion community studying turbulence-chemistry interactions due to their many advantages, including, the achievement of high Reynolds numbers, the realization of a range of combustion regimes from stable to local extinction/reignition, and the fact that they are more compact than jet flames [1-3]. The main motivation of computational studies on TCFs has been to test the underlying models for their validity and accuracy across a broad range of flame conditions [4-7].

Recently, we successfully applied the large-eddy simulation/probability density function (LES/PDF) methodology [8-10] to study turbulent premixed flames of the Yale TCF burner under various conditions that were previously studied experimentally $[11,12]$. The turbulence-chemistry interactions of the turbulent premixed flame with the counterflowing hot stoichiometric combustion products were investigated by comparing revealing conditional statistics from the simulations to those from the experiments. We found excellent agreement between the LES/PDF simulations and experiments for different flame conditions ranging from fully burning to nearly extinguished [12]. Furthermore, we investigated the LES/PDF equations in the direct numerical simulation (DNS) limit [13] by considering two laminar premixed flames. It is shown in [12] that when the conclusions of this DNS limit study are applied to the 3D LES/PDF simulations of the turbulent premixed counterflow flames, the calculated flame speed is close to the corresponding laminar flame speed for the observed mixing rate. This observation likely explains the success of the models.

In this present work, we consider two contrasting turbulent premixed flames from this LES/PDF computational study that are very different, qualitatively and quantitatively, to investigate the extinction/reignition events in more detail. The first flame is a high-burning (HB) flame with a low probability of extinction whereas the second one is a low-burning (LB) flame with a high probability of extinction. The main aim of

\footnotetext{
* Corresponding author

Present address: CD-adapco, 21800 Haggerty Road, Suite 300, Northville, MI 48167, USA

Email address: rrt38@cornell.edu (Ranjith R. Tirunagari) 
the present work is to investigate the extent to which the compositional structure of the turbulent flames is related to that of strained laminar flames. This is achieved by examining the particle data on the centerline from the unsteady, 3D LES/PDF simulations and comparing the cell-mean profiles of temperature $T$ to the corresponding profiles from steady, 1D, strained, opposed-jet laminar flame calculations.

Previous laminar flame calculations in the opposed-jet configuration have been performed $[2,4,14,15]$ to shed light on the behavior of their turbulent counterparts. A similar approach to that employed in the current work was employed in the previous works $[2,4]$, wherein the scatter plots of species and temperature from the experiments of turbulent partially premixed methane/air flame are compared to laminar flame profiles at different strain rates. The conditional mean of the experimental data from the scatter plots of species and temperature is well represented by a strained laminar flamelet. It is also observed that close to extinction, the measured scatter is closer to the laminar flamelet with the extinction strain rate.

The remainder of the paper is organized as follows. In Sec. 2, a brief description of the experimental study of the Yale TCF burner is provided, followed by a brief description of our previous LES/PDF computational study. We then draw attention to the two turbulent premixed flames studied in the present work. In Sec. 3, the focus is on differentiating the two turbulent premixed flames by analyzing the particle data from the LES/PDF simulations. The conclusions from the study are drawn in Sec. 4.

\section{Yale turbulent counterflow flame (TCF) burner in the premixed mode}

\subsection{Yale/Sandia experimental study}

The experimental configuration [11] consists of two coaxial opposed nozzles placed at a distance $d$ apart. The top stream is a fresh, cold reactants stream of homogenous premixed $\mathrm{CH}_{4} / \mathrm{O}_{2} / \mathrm{N}_{2}$ mixture with equivalence ratio $\phi_{u}$ at turbulent Reynolds number $R e_{t}$ and unburnt temperature $T_{u}=294 \mathrm{~K}$. The bottom stream consists of hot stoichiometric combustion products with measured temperature $T_{b}$, which is below the adiabatic flame temperature. The turbulent reactants stream then interacts with the counterflowing hot stoichiometric product stream to form a turbulent premixed counterflow flame propagating into the reactants stream. In the experiments, the high-turbulence levels in the reactants stream is generated by placing a turbulence generating plate (TGP) [16] inside the top nozzle, whereas it is omitted in the bottom nozzle. The bulk velocity $U_{b u l k}$ in the top stream is $11.2 \mathrm{~m} / \mathrm{s}$, which results in a bulk strain rate $K_{b u l k}\left(=2 U_{b u l k} / d\right)$ of $1400 s^{-1}$.

A parametric study is conducted to study the interactions of turbulent premixed flames with stoichiometric counterflowing hot combustion products by systematically varying the four identified parameters, 
i.e., $\phi_{u}, R e_{t}, T_{b}$ and $K_{b u l k}$. The instantaneous profiles of $\mathrm{OH}$ mass fraction $Y_{O H}$ (from the OH-LIF signal in the experiments) are used to identify the fresh product layer thickness $\Delta_{f}$ in which a binary progress variable $c$ is taken to be 1 . The first peak in the $\left|\nabla Y_{O H}\right|$ profile (while traversing the profile from the products stream to the reactants stream) is defined as the gas mixing layer interface (GMLI). The GMLI represents the interface between the two counterflowing streams. The fresh product layer thickness $\Delta_{f}$ represents the distance between the end of flame region and the GMLI. The conditional mean progress variable $\langle c \mid \Delta\rangle$ and the PDF of fresh product layer thickness $\Delta_{f}$ are used to quantitatively study the effects of the critical parameters, where $\Delta$ is the distance measured from the GMLI. It is important to note that $\langle c \mid \Delta\rangle$ represents the probability of finding fresh combustion products from the premixed flame at a distance $\Delta$ from the GMLI, and therefore $1-\langle c \mid \Delta=0\rangle$ represents the probability of localized extinction at the GMLI. More details about the experimental study can be found in [11].

In both of the flames considered in this work, the upper stream is a homogeneous, lean, unburnt fuel/air mixture, whereas the lower stream consists of burnt stoichiometric products. As in the experimental study [11] we refer to these flames as premixed, whereas in some terminologies they would be called partially premixed. Because the mass fraction of $N_{2}$ is different in the two streams (and $N_{2}$ is taken to be inert) we can use this mass fraction to define a mixture fraction, which is zero in the reactants and unity in the products. This mixture fraction obviously does not have the same significance that it does in non-premixed combustion.

\subsection{LES/PDF computational study}

In recent work [12], we studied the turbulent premixed flames described in Sec. 2.1 using the LES/PDF computational methodology [8-10]. The low-Mach number, variable-density Navier-Stokes equation solver, NGA [17], is employed to solve the filtered LES transport equations for mass and momentum on a structured grid in cylindrical coordinates. The Lagrangian dynamic sub-grid scale model [18] is used to obtain the turbulent viscosity and diffusivity. The particle/mesh code, HPDF [19-21], is employed to evolve the position and composition of many computational particles by solving a set of stochastic differential equations. Molecular transport is modeled as a random walk term in the stochastic differential equation for the particle position. The classical interaction by exchange with the mean (IEM) mixing model [22] is employed to represent mixing in the differential equation for the particle composition. The value of the mixing model constant, $C_{m}$, is 4 . The normalized mixing rate, $\Omega_{R} \tau_{L}$, (normalized by the laminar-flame time scale $\tau_{L}$ ), is found to be around 20 for these flames. More details can also be found in [12]. A 16-species augmented reduced mechanism for methane oxidation is used for the chemical mechanism [23]. 
The computational domain is taken as the cylindrical volume between the two nozzle exit planes. The specification of velocity boundary conditions at the inflows of the computational domain (i.e., at the nozzle exit planes) is non-trivial. The inflow methodology described in [24] is employed to match the turbulent Reynolds number $R e_{t}$ in the simulations to that of the experiments. A total of approximately 0.3M LES grid cells and $6 \mathrm{M}$ particles (where $1 \mathrm{M}=10^{6}$ ) are used in the LES/PDF simulations. The normalized grid spacing, $h / \delta_{L}$, (normalized by laminar flame thickness $\delta_{L}$ ), is found to yield calculated flame thickness twice the laminar value as the grid is coarser by about a factor of 4 compared to that required to resolve the laminar flame thickness. However, the calculated flame speed is found to be close to the laminar flame speed. The same approach as employed in the experimental study for extracting the conditional statistics is used in the computational study. The simulation results are found to be in excellent agreement with the experimental data for the two key quantities, i.e., $\langle c \mid \Delta\rangle$ and the $\mathrm{PDF}$ of $\Delta_{f}$, for all the flame conditions of the parametric study. More details about the computational study and comparisons of the simulation results to the experiments can be found in [12].

\subsection{Flames considered}

In this present work, we consider two contrasting turbulent premixed flames from the computational study of Sec. 2.2 to investigate the extinction/reignition events in more detail. The two flames considered are:

1. High-burning (HB) flame with $\phi_{u}=0.85$ and $T_{b}=1850 \mathrm{~K}$.

2. Low-burning (LB) flame with $\phi_{u}=0.7$ and $T_{b}=1800 \mathrm{~K}$.

The other two parameters, namely, $K_{b u l k}$ and $R e_{t}$ have the values of $1400 \mathrm{~s}^{-1}$ and 1050 , respectively, for both flames.

\section{Results from the LES/PDF simulations}

\subsection{Qualitative analysis}

Figure 1 shows the visualization of the two turbulent premixed flames sandwiched between the nozzles using the contour plots of temperature $T$ on two perpendicular planes intersecting the solution domain through the center. The top row in Fig. 1 shows the HB flame at different instants of increasing time, for one of the relatively rare local extinction events. The bottom row in Fig. 1 shows the LB flame at different instants of increasing time, for a common local extinction event. Clearly, the LB flame burns less vigorously when compared to the HB flame. 


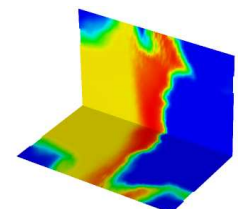

(a)

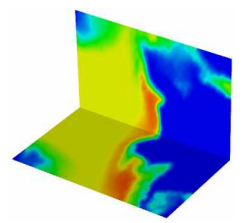

(g)

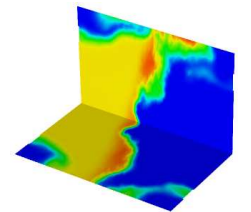

(b)

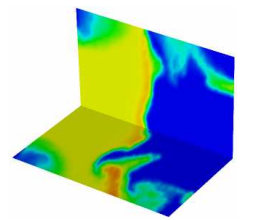

(h)

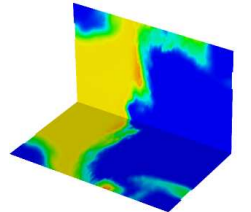

(c)

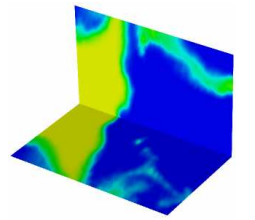

(i)

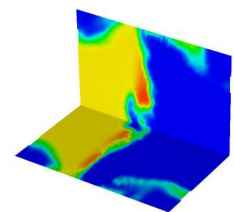

(d)

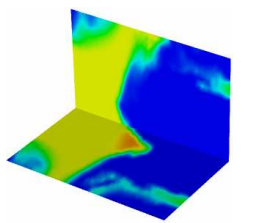

(j)

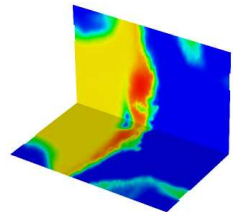

(e)

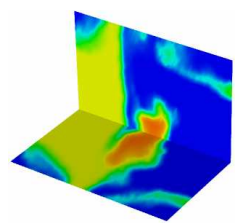

$(\mathrm{k})$

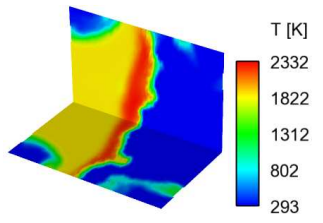

(f)

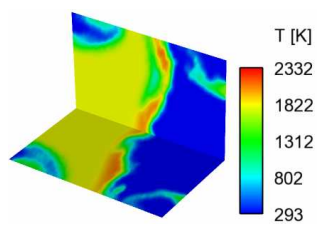

(1)

Figure 1: Instantaneous contour plots of temperature from the LES/PDF simulations of the HB (top row) and LB (bottom row) flames on two perpendicular planes intersecting the solution domain through the center. The products nozzle is at the left and the reactants nozzle at the right. The top frames are taken at time $t=17.3,18.0,18.6,18.8,19.0$, and $19.3 \mathrm{~ms}$, respectively. The bottom frames are taken at time $t=25.7,26.0,29.6,37.2,37.8$, and $38.6 \mathrm{~ms}$, respectively.

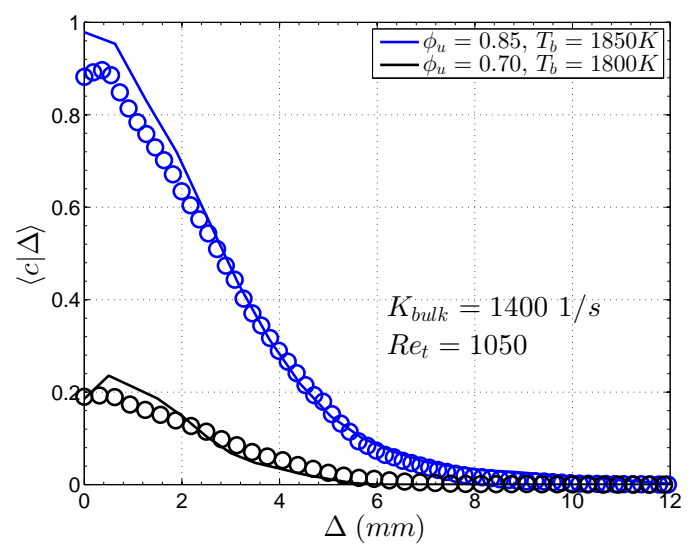

Figure 2: Conditional mean progress variable $\langle c \mid \Delta\rangle$ as a function of distance $\Delta$ from the GMLI for the HB (blue) and LB (black) flames; lines: LES/PDF simulations, symbols: experimental data [11].

\subsection{Conditional statistics}

Figure 2 shows the conditional mean progress variable $\langle c \mid \Delta\rangle$ as a function of distance $\Delta$ from the GMLI for the HB and LB flames. The HB flame has a lower extinction probability of $10 \%$ at the GMLI whereas the LB flame has a much higher value of $80 \%$.

\subsection{Strained opposed-jet laminar flames}

To study the response of laminar premixed flames to varying strain rates, two separate sets of calculations are performed in CHEMKIN-PRO [25] for one-dimensional, strained, opposed-jet, laminar premixed flames with the stream compositions the same as those of the two studied flames (HB and LB). The chemical mechanism 


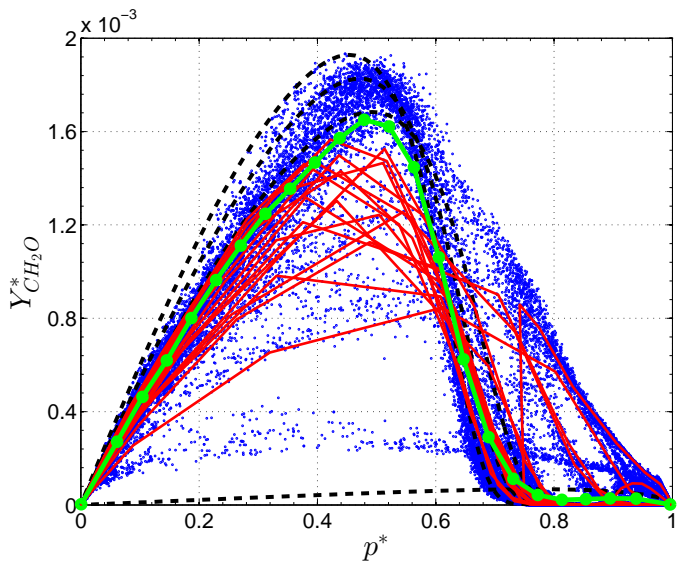

(a)

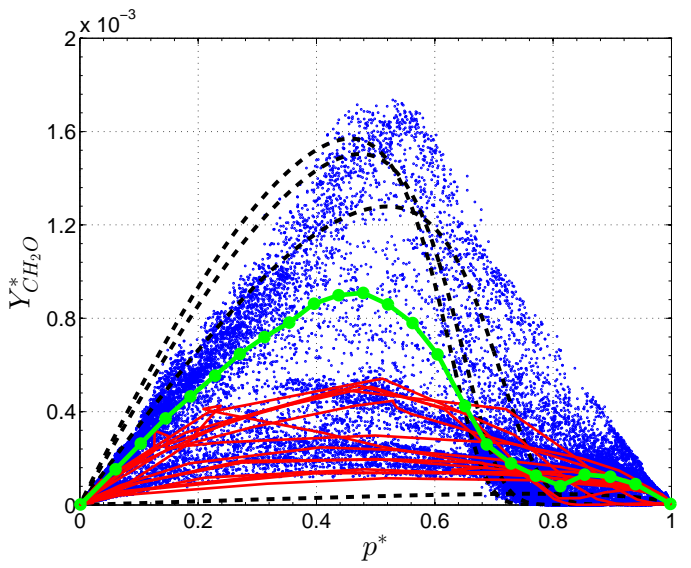

(b)

Figure 3: From the LES/PDF simulations, scatter plots of particle $\mathrm{CH}_{2} \mathrm{O}$ species mass fraction, $\mathrm{Y}_{\mathrm{CH}}^{*} \mathrm{O}$, vs. progress variable $p^{*}$ on the centerline for the (a) HB and (b) LB flames for 20 randomly chosen samples. In each plot, the solid red line is the cell-mean profile of each sample, the solid green line with circles is the conditional mean of $Y_{\mathrm{CH}}^{*} \mathrm{O}$ conditioned on $p^{*}$ and the black dashed lines are the laminar profiles (Sec. 3.3). The lower strain rates have higher values of peak $Y_{\mathrm{CH}_{2} \mathrm{O}}$ and the flat, black dashed line is the extinguished profile.

used is GRI-Mech version 1.2 [26]. As in the LES/PDF calculations, the molecular diffusivities of species are taken to be same and equal to the thermal diffusivity under the unity Lewis number assumption. In the laminar flame calculations, the strain rate is evaluated using the same equation as that used for the bulk strain rate $K_{\text {bulk }}$ (see Sec. 2.1). The strain rate is varied by varying the bulk velocities in the two streams. For the HB flame, the strain rate is varied from 375 to $2687.5 \mathrm{~s}^{-1}$ with extinction occurring at a strain rate of $S_{\text {ext }}=2650 \mathrm{~s}^{-1}$. For the LB flame, the strain rate is varied from 250 to $2125 \mathrm{~s}^{-1}$ and has a lower value of extinction strain rate with $S_{\text {ext }}=2000 \mathrm{~s}^{-1}$. The laminar profiles for varying strain rates are presented in Sec. 3.4.

\subsection{Scatter plots}

Figure 3 shows the scatter plots of particle $\mathrm{CH}_{2} \mathrm{O}$ mass fraction, $\mathrm{Y}_{\mathrm{CH}}^{*} \mathrm{O}$, vs. progress variable $p^{*}$ on the centerline for the two flames. Note that the superscript '*' denotes that the quantities are evaluated at the particle position. The progress variable $p$ is defined as

$$
p=\frac{Y_{\mathrm{CO}_{2}}+Y_{\mathrm{H}_{2} \mathrm{O}}}{\max \left(Y_{\mathrm{CO}_{2}}+Y_{\mathrm{H}_{2} \mathrm{O}}\right)},
$$

where $\mathrm{Y}_{\mathrm{CO}_{2}}$ and $\mathrm{Y}_{\mathrm{H}_{2} \mathrm{O}}$ are the mass fractions of $\mathrm{CO}_{2}$ and $\mathrm{H}_{2} \mathrm{O}$, respectively. Hence, the value of $p$ is zero in the reactants stream and unity in the products stream. As can be seen from the figure, the two scatter 
plots are quite different. The cell-mean profiles (solid red lines) for the HB flame are closer to the laminar profiles (black dashed lines) at lower strain rates whereas the cell-mean profiles of the LB flame are closer to the flat, black dashed line corresponding to the extinguished profile. As a result, the conditional mean (solid green line with circles) of $Y_{\mathrm{CH}_{2} \mathrm{O}}^{*}$ conditioned on $p^{*},\left\langle Y_{\mathrm{C} \mathrm{H}_{2} \mathrm{O}}^{*} \mid p^{*}\right\rangle$, is different for the two flames with the peak of the conditional mean higher for the HB flame when compared to that of the LB flame. We also observe the mixing line between the peak of $Y_{\mathrm{CH}_{2} \mathrm{O}}$ and the products stream, especially in the $\mathrm{HB}$ case, and it is above the laminar solution.

We now define the mixture fraction $\xi$ based on the mass fraction of $N_{2}, Y_{N_{2}}$, as follows:

$$
\xi=\frac{Y_{N_{2}}-Y_{N_{2}, \min }}{Y_{N_{2}, \max }-Y_{N_{2}, \min }},
$$

where $Y_{N_{2}, \min }=0.6274$ and 0.6347 are the minimum values of $Y_{N_{2}}$, which occur in the reactants stream, and $Y_{N_{2}, \max }=0.6658$ and 0.7009 are the maximum values, which occur in the products stream of the HB and LB flames, respectively. Hence, $\xi$ is zero in the reactants stream and unity in the products stream. (Since equal diffusivities are used, the mixture fractions based on each element $(C, H, O, N)$ are the same and equal to $\xi$, since $N_{2}$ is taken to be inert.) The mixture fraction in this study signifies the amount of molecular mixing between the two streams.

A normalized distance $Z$ is then defined based on the mixture fraction $\xi$ as follows:

$$
Z=\operatorname{erfc}^{-1}(2 \xi)
$$

where $\operatorname{erfc}^{-1}$ is the inverse of the complementary error function. The reason for using $Z$ (instead of $\xi$ ) in plots below is that it stretches the region close to $\xi=0(Z \rightarrow \infty)$ where mildly-strained partially-premixed flames are located. (Note that, for a diffusive layer with constant diffusivity $D$ and strain rate $K$, the distance through the layer is proportional to $Z(D / K)^{1 / 2}$.)

Figure 4 shows the scatter plots of particle temperature $T^{*} v s$. particle normalized distance $Z^{*}$ on the centerline, color-coded by the particle $\mathrm{OH}$ mass fraction $Y_{O H}^{*}$. The left and right columns show the scatter plots for the HB and LB flames, respectively. The scatter plots in the first row are plotted for the same 20 samples shown in Fig. 3. The cell-mean profiles from the particle data at the same time instants are shown as black dashed lines. The magenta lines are the laminar profiles from Sec. 3.3 with strain rate increasing from right to left in the plots. The leftmost magenta line shows the laminar profile for the extinguished case. 


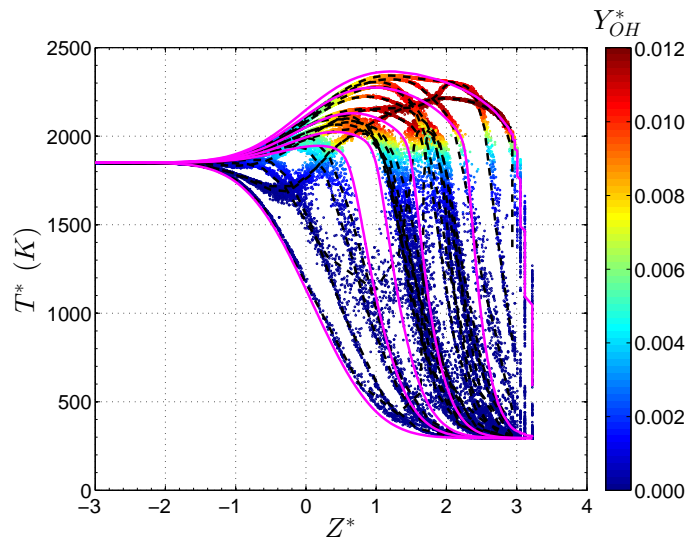

(a)

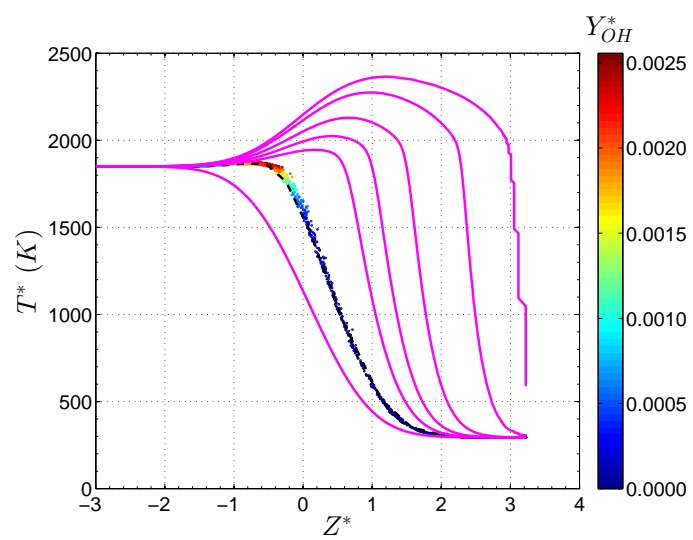

(c)

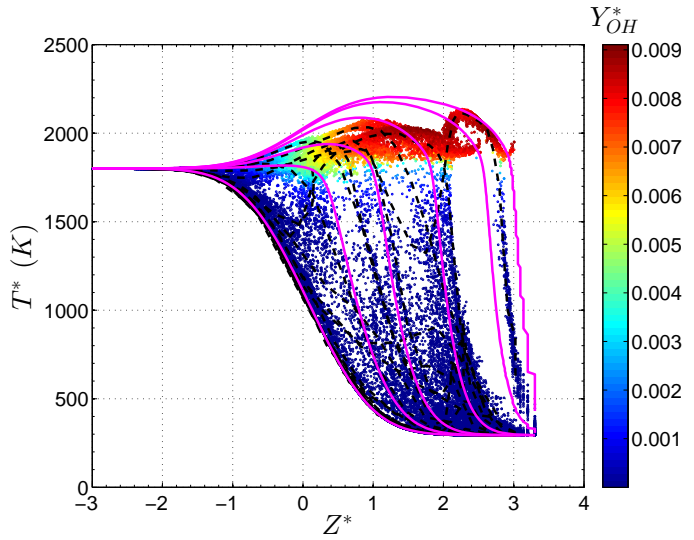

(b)

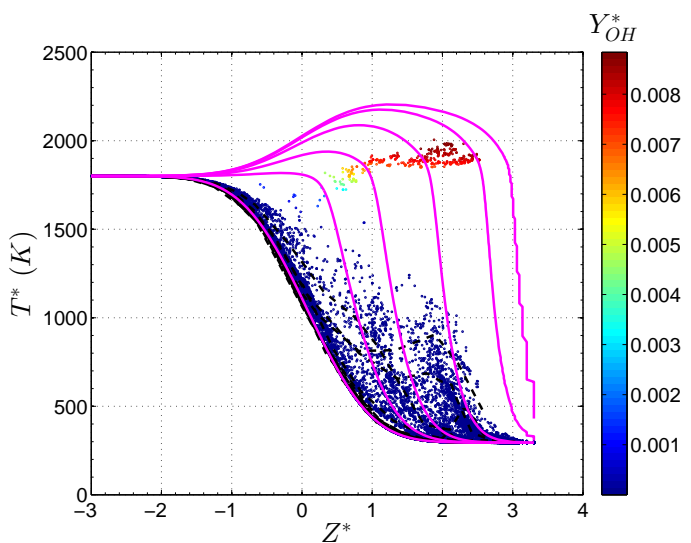

(d)

Figure 4: From the LES/PDF simulations, scatter plots of particle temperature $T^{*} v s$. normalized distance $Z^{*}$ on the centerline, color-coded by $\mathrm{OH}$ species mass fraction $Y_{O H}^{*}$. The left and right columns are for the HB and LB flames, respectively. The top row shows the 20 chosen samples. The bottom row shows the subset of the 20 samples that are extinguished. In each plot, the black dashed line is the cell-mean profile of each sample and the solid magenta lines are the laminar profiles from the study of Sec. 3.3 in the increasing order of strain rate from right to left. The values of strain rates used in the laminar flame calculations for the HB flame are $375,750,1400,2250,2650$, and $2687.5 \mathrm{~s}^{-1}$. The values for the LB flame are 250, 375, 750, $1400,2062.5$, and $2125 \mathrm{~s}^{-1}$.

The shift of the laminar profiles from right to left as the strain rate is increased can be understood through the following rough argument. A premixed laminar flame with flame speed $s_{L}$ (assumed to be independent of the strain rate $K$ ) propagates a distance $z$ into the reactants such that $s_{L}=K z$, i.e., the incoming flow velocity equals the laminar flame speed. Hence, the normalized distance is

$$
Z=z\left(\frac{K}{D}\right)^{1 / 2}=\frac{s_{L}}{(K D)^{1 / 2}},
$$

so that the profile of $T$ vs. $Z$ moves to the left (i.e., to smaller values of $Z$ ) as $K$ increases. For the extinguished flame, if the diffusivity were uniform, $T(Z)$ would be the shape of the complimentary error 
function, centered at $Z=0$.

It can be inferred from the plots that the cell-mean profiles of $T$ generally look quite similar to the laminar profiles at different strain rates. It is emphasized, however, that the LES/PDF simulations are non-trivially 3D and unsteady, and indeed some of the profiles have multiple temperature maxima.

A cutoff value equal to ten times the value of $Y_{O H}$ in the products stream is used to distinguish a burning sample from an extinguished sample. If the maximum of the cell-mean profile of $Y_{O H}$ is below this cutoff value, then the sample is considered to be extinguished.

It can be inferred from Fig. 4(a) that the HB flame has more samples away from the extinguished laminar profile and the sample density tends towards lower strain rates. In contrast, Fig. 4(b) shows that the LB flame has more samples close to the extinguished laminar profile as can be seen with the increased sample density and hence, has a high value of probability of local extinction. Further, the extinguished samples (bottom row) are closer to the extinguished laminar profiles with more samples present for the LB case (12 samples) as compared to that of the HB flame (1 sample).

\subsection{Equivalent steady strain rate (ESSR)}

Let $\hat{T}(Z, K)$ denote the temperature as a function of $Z$ for the laminar flame with strain rate $K$. We define $T_{o}=1250 \mathrm{~K}$ as a reference temperature, and for a burning flame we define $Z_{o}$ to be the value of $Z$ at which the cell-mean temperature equals $T_{o}$. Then the "equivalent steady strain rate" (ESSR) $S$ is defined implicitly by $\hat{T}\left(Z_{o}, S\right)=T_{o}$. That is, the turbulent flame and the laminar flame with strain rate $S$ both have temperature $T_{o}$ at $Z=Z_{o}$. The laminar flame is extinguished for $K \geq S_{\text {ext }}$, and we define $Z_{\text {ext }}$ implicitly by $T_{o}=\hat{T}\left(Z_{e x t}, S_{e x t}\right)$. A turbulent flame with $Z_{o} \leq Z_{\text {ext }}$ is deemed to be extinguished, and $S$ is defined to be $S_{\text {ext }}$.

Figure 5 shows the empirical cumulative distribution function (CDF) of the ESSR $S$, conditional on $S<S_{\text {ext }}$, for the two flames. It can be seen that the conditional CDFs of $S$ for the two flames are somewhat similar, but not convincingly the same. There is a broad, almost flat distribution, with no discernible concentration around the bulk strain rate $K_{b u l k}$.

Table 1 shows the extinction probabilities of the HB and LB flames using three different definitions of an extinguished sample - (i) the GMLI method (see Sec. 3.2), (ii) the cutoff value $Y_{O H, c u t}$ equal to ten times the value of $Y_{O H}$ in the products stream (see Sec. 3.4), and (iii) the number of samples for which $Z_{o} \leq Z_{\text {ext }}$ using the ESSR method. As may be seen, all three criteria clearly distinguish between low and high probabilities of extinction in the two flames, with the differences between them being of order $\pm 8 \%$. 


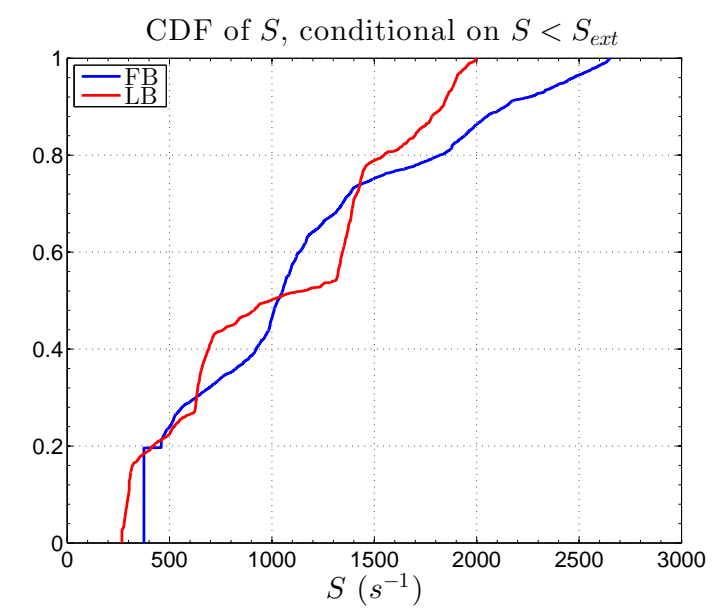

Figure 5: Empirical cumulative distribution function (CDF) of the ESSR $S$, conditional on $S<S_{\text {ext }}$, from the LES/PDF simulations of the HB (blue) and LB (red) flames. The extinction strain rates $S_{\text {ext }}$ for the HB and LB flames are 2650 and $2000 s^{-1}$, respectively.

Table 1: Extinction probabilities of the HB and LB flames.

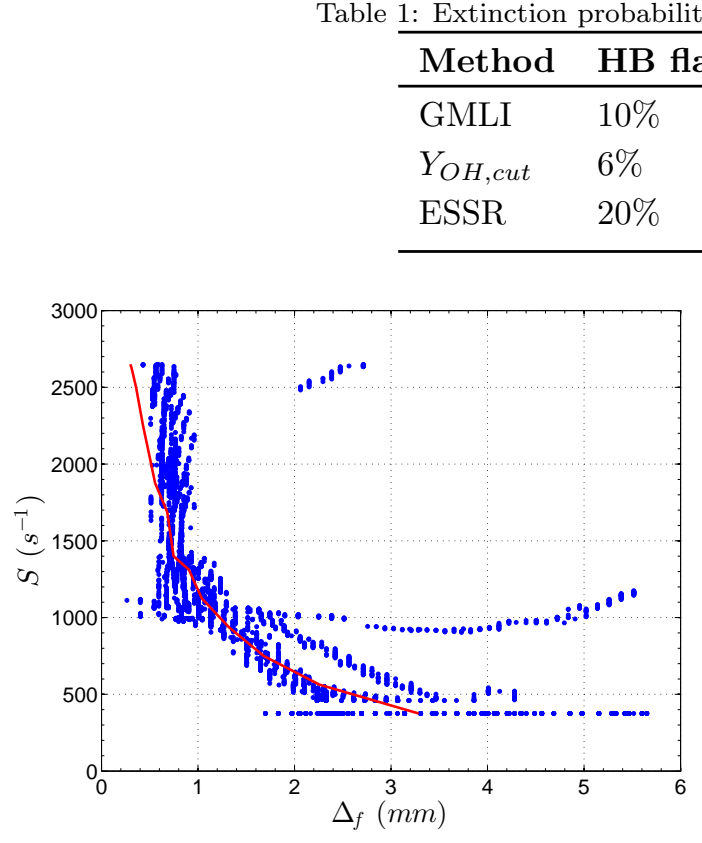

(a)

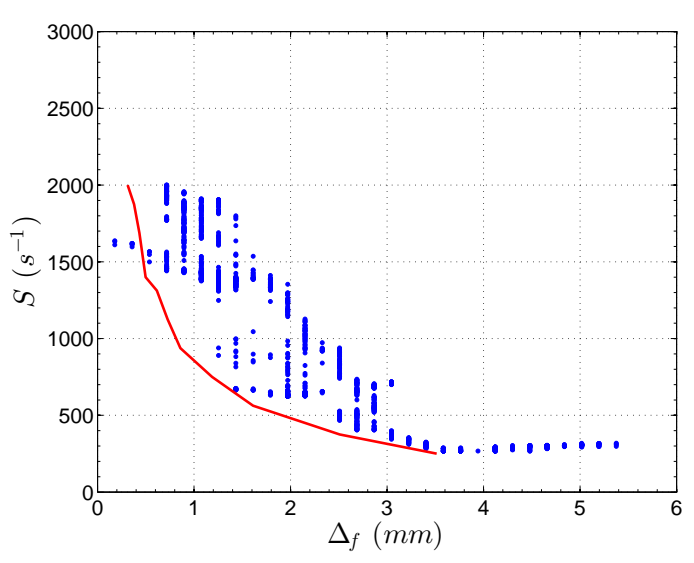

(b)

Figure 6: Scatter plots of the ESSR $S$ vs. fresh product layer thickness $\Delta_{f}$ for the burning samples $\left(S<S_{\text {ext }}\right)$ from the LES/PDF simulations of the (a) HB and (b) LB flames, respectively. The solid red line is the laminar profile from the study of Sec. 3.3.

Figures 6(a) and 6(b) show the scatter plots of the equivalent steady strain rate $S$ vs. the fresh product layer thickness $\Delta_{f}$ from the GMLI method for the burning samples (i.e., $S<S_{e x t}$ ) of the HB and LB flame, respectively. The red solid lines are the laminar profiles from the laminar study of Sec. 3.3. It can be inferred 
from the figures that as the value of the strain rate increases, the product layer thickness becomes thinner, as expected. Due to the difference in the extinction probabilities, the HB flame has more burning samples compared to the LB flame. The scatter for the HB flame follows the corresponding laminar profile closely; however, for the LB flame, the product layer thickness observed for a particular strain rate is thicker than that predicted by the laminar profile. We observe a horizontal band at $S=375 \mathrm{~s}^{-1}$ for the HB case. In fact, this band represents all ESSR values which are below $375 \mathrm{~s}^{-1}$ as it is difficult to determine the ESSR value from interpolation when $S<375 \mathrm{~s}^{-1}$ due to the asymptotic limits of the complementary error function. The two other horizontal bands around $S=1000$ and $2500 \mathrm{~s}^{-1}$ occur for a certain type of the profile of centerline $\mathrm{OH}$ mass fraction, $Y_{O H}$. These bands occur when there are multiple crossings of the $\mathrm{OH}$ profile for a given cutoff value of $Y_{O H}$ while determining the product layer thickness. Therefore, for such cases, the estimate of $\Delta_{f}$ is higher. On the other hand, the ESSR is calculated based on the temperature condition, $T_{o}=1250 \mathrm{~K}$.

\section{Conclusions}

The following conclusions can be drawn:

1. Two contrasting turbulent premixed (or partially-premixed) counterflow flames that have different probabilities of extinction are studied using the LES/PDF methodology to examine extinction/reignition events.

2. One-dimensional, steady, strained, opposed-jet laminar flame calculations are performed to investigate the effects of strain rates on their laminar counterparts and to find the extinction strain rates $S_{\text {ext }}$ for the HB and LB flames.

3. The scatter plots from the particle data of (a) $\mathrm{CH}_{2} \mathrm{O}$ mass fraction $\mathrm{Y}_{\mathrm{CH}_{2} \mathrm{O}}^{*}$ vs. progress variable $p^{*}$, and (b) temperature $T^{*} v$ s. normalized distance $Z^{*}$ for the two flames are quite different with more samples close to the extinguished laminar profile for the LB flame. Additionally, the cell-mean profiles of $T$ vs. $Z$ generally look quite similar to the laminar profiles at different strain rates although the LES/PDF simulations are non-trivially 3D and unsteady.

4. The instantaneous equivalent steady strain rates (ESSRs) for the two flames are calculated using the cell-mean profiles of temperature $T$ vs. the normalized distance $Z$. The CDF of $S$, conditional on $S<S_{\text {ext }}$, for the two flames are somewhat similar, with the distributions being broad without a peak close to the bulk strain rate $K_{b u l k}$. 
5. The probabilities of extinction calculated for the two flames using the distribution of the ESSR compare well with the corresponding probabilities evaluated using the GMLI method. This confirms the validity of the experimental approach based solely on $\mathrm{OH}$ mass fraction.

6. The scatter plots of the ESSR $S$ vs. fresh product layer thickness $\Delta_{f}$ for the burning samples $\left(S<S_{\text {ext }}\right)$ reveal that the increase in strain rate leads to a decrease in the product layer thickness. The scatter for the HB flame follow the laminar profile quite closely, whereas the thicknesses observed for the LB flame for a particular strain rate are higher compared to its laminar prediction.

\section{Acknowledgements}

The authors thank Dr. B. Coriton for helping with the experimental data and Dr. M. W. A. Pettit for helping with the inflow boundary conditions using the 'PsiPhi' code. We gratefully acknowledge the California Institute of Technology, the University of Colorado at Boulder and Stanford University for licensing the NGA code used in this work. The authors thank the University of Tennessee and Oak Ridge National Laboratory Joint Institute for Computational Sciences and Cornell University Center for Advanced Computing for providing the computational resources required to perform this work.

This research is funded by the U.S. Department of Energy, Office of Science, Office of Basic Energy Sciences under award number DE-FG02-90 ER14128. This work used the Extreme Science and Engineering Discovery Environment (XSEDE), which is supported by National Science Foundation grant number ACI1053575 .

\section{References}

[1] G. Coppola, B. Coriton, A. Gomez, Highly turbulent counterflow flames: A laboratory scale benchmark for practical systems, Combust. Flame 156 (2009) 1834-1843.

[2] D. Geyer, A. Kempf, A. Dreizler, J. Janicka, Turbulent opposed-jet flames: A critical benchmark experiment for combustion LES, Combust. Flame 143 (2005) 524-548.

[3] K. H. H. Goh, P. Geipel, R. P. Lindstedt, Lean premixed opposed jet flames in fractal grid generated multiscale turbulence, Combust. Flame 161 (2014) 2419-2434.

[4] D. Geyer, A. Dreizler, J. Janicka, A. D. Permana, J. Y. Chen, Finite-rate chemistry effects in turbulent opposed flows: comparison of Raman/Rayleigh measurements and Monte Carlo PDF simulations, Proc. Combust. Inst. 30 (2005) $711-718$.

[5] A. Kempf, H. Forkel, J. Y. Chen, A. Sadiki, J. Janicka, Large-eddy simulation of a counterflow configuration with and without combustion, Proc. Combust. Inst. 28 (2000) 35-40.

[6] R. P. Lindstedt, D. S. Luff, J. H. Whitelaw, Velocity fields of fuel lean premixed turbulent opposed jet flames, Proc. Combust. Inst. 31 (2007) 1459-1466. 
[7] M. W. A. Pettit, B. Coriton, A. Gomez, A. M. Kempf, Large-eddy simulation and experiments on non-premixed highly turbulent opposed jet flows, Proc. Combust. Inst. 33 (2011) 1391-1399.

[8] S. B. Pope, Turbulent Flows, Cambridge University Press, Cambridge, 2000.

[9] H. Pitsch, Large-eddy simulation of turbulent combustion, Annu. Rev. Fluid. Mech. 38 (2006) 453-482.

[10] S. B. Pope, PDF methods for turbulent reactive flows, Prog. Energy Combust. Sci. 11 (1985) 119-192.

[11] B. Coriton, J. H. Frank, A. Gomez, Effects of strain rate, turbulence, reactant stoichiometry and heat losses on the interaction of turbulent premixed flames with stoichiometric counterflowing combustion products, Combust. Flame 160 (2013) 2442-2456.

[12] R. R. Tirunagari, S. B. Pope, An investigation of turbulent premixed counterflow flames using large-eddy simulations and probability density function methods, Combust. Flame, 166 (2016) 229-242.

[13] R. R. Tirunagari, S. B. Pope, LES/PDF for premixed combustion in the DNS limit, Combust. Theor. Model., 2016, in press, (DOI: 10.1080/13647830.2016.1188991). Preprint available at https://tcg.mae.cornell.edu/pubs/Tirunagari_P_CTM_2015.pdf.

[14] B. Coriton, J. H. Frank, A. G. Hsu, M. D. Smooke, A. Gomez, Effect of quenching of the oxidation layer in highly turbulent counterflow premixed flames, Proc. Combust. Inst. 33 (2011) 1647-1654.

[15] B. Coriton, M. D. Smooke, A. Gomez, Effect of the composition of the hot product stream in the quasi-steady extinction of strained premixed flames, Combust. Flame 157 (2010) 2155-2164.

[16] G. Coppola, A. Gomez, Experimental investigation on a turbulence generation system with high-blockage plates, Exp. Therm. Fluid Sci. 33 (2009) 1037-1048.

[17] O. Desjardins, G. Blanquart, G. Balarac, H. Pitsch, High order conservative finite difference scheme for variable density low mach number turbulent flows, J. Comput. Phys. 227 (2008) 7125-7159.

[18] C. Meneveau, T. S. Lund, W. H. Cabot, A lagrangian dynamic subgrid-scale model of turbulence, J. Fluid Mech. 319 (1996) 353-385.

[19] H. Wang, S. B. Pope, Large-eddy simulation/probability density function modeling of a turbulent $\mathrm{CH}_{4} / \mathrm{H}_{2} / \mathrm{N}_{2}$ jet flame, Proc. Combust. Inst. 33 (2011) 1319-1330.

[20] Y. Yang, H. Wang, S. B. Pope, J. H. Chen, Large-eddy simulation/probability density function modeling of a non-premixed $\mathrm{CO} / \mathrm{H}_{2}$ temporally evolving jet flame, Proc. Combust. Inst. 34 (2013) 1241-1249.

[21] P. P. Popov, H. Wang, S. B. Pope, Specific volume coupling and convergence properties in hybrid particle/finite volume algorithms for turbulent reactive flows, J. Comput. Phys. 294 (2015) 110-126.

[22] J. Villermaux, J. C. Devillon, Représentation de la coalescence et de la redispersion des domaines de ségrégation dans un fluide par un modèle d'interaction phénoménologique, in: Proc. 2nd Int. Symp. Chem. React. Eng., Elsevier, New York, 1972, pp. 1-13.

[23] C. J. Sung, C. K. Law, J. Y. Chen, An augmented reduced mechanism for methane oxidation with comprehensive global parametric validation, Proc. Combust. Inst. 27 (1998) 295-304.

[24] R. R. Tirunagari, M. W. A. Pettit, A. M. Kempf, S. B. Pope, A simple approach for specifying velocity inflow boundary conditions in simulations of turbulent opposed-jet flows, Flow Turbul. Combust., 2016, in press, (DOI: 10.1007/s10494016-9743-4). Preprint available at https://tcg.mae.cornell.edu/pubs/Tirunagari_PKP_FTC_2015.pdf.

[25] R. J. Kee, F. M. Rupley, J. A. Miller, M. E. Coltrin, J. F. Grcar, E. Meeks, H. K. Moffat, A. E. Lutz, G. Dixon-Lewis, M. D. Smooke, J. Warnatz, G. H. Evans, R. S. Larson, R. E. Mitchell, L. R. Petzold, W. C. Reynolds, M. Caracotsios, 
W. E. Stewart, P. Glarborg, C. Wang, O. Adigun, Chemkin collection, release 3.6, in: Reaction Design, Inc., San Diego, CA, 2000 .

[26] M. Frenklach, H. Wang, M. Goldenberg, G. P. Smith, D. M. Golden, C. T. Bowman, R. K. Hanson, W. C. Gardiner, V. Lissianski, GRI-Mech—an optimized detailed chemical reaction mechanism for methane combustion, Tech. rep., Report No. GRI-95/0058, available at http://www.me.berkeley.edu/gri_mech/ (1995). 